Research Article

\title{
Wave Dispersion and Propagation in Linear Peridynamic Media
}

\author{
Xiaolong Zhang $\mathbb{D}$, Zhenying Xu $\mathbb{D}$, and Qing Yang \\ School of Mechanical Engineering, Jiangsu University, Zhenjiang 212013, China \\ Correspondence should be addressed to Zhenying Xu; zhyxu77@163.com
}

Received 25 December 2018; Revised 18 April 2019; Accepted 13 May 2019; Published 9 June 2019

Academic Editor: Toshiaki Natsuki

Copyright (C) 2019 Xiaolong Zhang et al. This is an open access article distributed under the Creative Commons Attribution License, which permits unrestricted use, distribution, and reproduction in any medium, provided the original work is properly cited.

\begin{abstract}
We detail the linear peridynamic wave equation with a nonlocal integral form based on the linear peridynamic and dynamic theory. Wave dispersion in an infinite maraging steel material is obtained by analyzing the linear peridynamic wave equation. The dispersion curves, group velocity, phase velocity, and other wave parameters of the shear and longitudinal waves in an infinite media are obtained using numerical methods. We obtained the optimal calculation parameters by analyzing the weight function, horizon, mesh size, and other numerical calculation parameters on the dispersion curve. We simulated the propagation of waves in an infinite media by applying these parameters into the peridynamic wave equation. We conclude that the wave model can generate waves that propagate in all directions with initial loads. The wavefront is an ellipsoid.
\end{abstract}

\section{Introduction}

Ultrasonic waves with unidirectionality and penetration properties are widely used in nondestructive testing [1-4]. In order to study the propagation of ultrasonic waves, the community usually uses finite element analysis methods to solve elastic dynamic equations in continuous media [5-8]. However, the derivative function does not exist at the defect when there is a defect (discontinuity) in the media [3, 4]. It is necessary to remesh the grid to make the defect outside of the boundary [9]. This method can simulate ultrasonic wave propagation in a media with the defect; however, the stresses at the crack tip are mathematically singular because of their undefined spatial derivatives. To study ultrasonic wave propagation in the media, we used the integral equation to describe the wave displacement change with location and time-named "the peridynamic method" $[10,11]$. This method falls into the category of nonlocal models $[12,13]$ because particles separated by a finite distance can interact with each other $[14,15]$. The peridynamic theory of solid mechanics has been proposed as a means of treating discontinuous media via a mathematical model that does not require a smooth distribution of mass or differentiability of the deformation [10]. The starting point of the theory is that the internal forces acting on a material point are determined via interactions between the point and all others within a finite distance from it. The peridynamic model uses integrals rather than differential equations so that the mathematical structure does not break down when a discontinuity occurs. Rather, the fracture is treated as a natural outcome of deformation that emerges according to the equations of motion and the constitutive model [16].

The theory of peridynamic waves was first proposed by Silling [10] in 2000, with a study of the propagation of linear stress waves and a discussion of wave dispersion. In 2003, Silling et al. [17] discussed the propagation of waves in a discontinuities bar. Later, Zimmermann [18] explored many features of the peridynamic theory including certain aspects of wave motion, material stability, and numerical solution techniques. In 2007, Silling et al. [19] gave the relationship between stress, displacement, and location under small deformation conditions making it easier to solve the microelastic peridynamic wave equation. Silling [20] gave the linearization theory of peridynamic in 2010. This theory paves the way for the study of linear elastic waves, making the peridynamic wave equation easier to solve. Bažant et al. [21] systematically studied the peridynamic stress wave in 2016 and gave the bond-based peridynamic stress wave theory and dispersion analysis. In the same year, Silling [22] studied solitary waves in elastic media and found that the 
solitary wave velocity is greater than that of the elastic wave. Butt et al. [23] conducted a detailed study of the peridynamic stress wave under linear elastic conditions in 2017. They then analyzed the state-based peridynamic wave dispersion and studied the mesh size, horizon, and weight function influence on dispersion.

In this paper, we gave the wave propagation equation a nonlocal integral form based on the research of linear peridynamic theory. The dispersion matrix [10] of the wave is given from the linear peridynamic theory, and the dispersion of the longitudinal and shear waves in the infinite media is analyzed. The phase velocity and group velocity of the waves in the infinite peridynamic media are given. The effects of weight function, horizon, and mesh size on the dispersion are analyzed. The peridynamic simulation is used in the infinite media to analyze the difference between the shear wave and the longitudinal wave. By analyzing the wave model, we conclude that the wavefront of the single-point excited wave is an ellipsoid.

\section{Basic Equations of Linear Peridynamic Media}

The continuum peridynamic wave theory is in contrast to the classical continuum theory. Any material point $x$ in the reference configuration is acted upon by forces due to the deformation of all the material points $x^{\prime}$ within the neighborhood of finite radius $\delta$ centered at $x$. The radius $\delta$ is called the horizon, and the material points within this neighborhood of $x$ in the reference configuration are called the family of $x$.

A material point $x$ at time $t$ has a force density $b(x, t)$ acting on it, and the motion is given by the following equation [24]:

$$
\begin{aligned}
\rho(x) \ddot{u}(x, t)= & L_{u}(x, t)+b(x, t), \quad \forall x \in \beta, t \geq 0, \\
L_{u}(x, t)= & \int_{\beta}\left\{\underline{T}[x, t]\left\langle x^{\prime}-x\right\rangle-\underline{T}\left[x^{\prime}, t\right]\right. \\
& \left.\cdot\left\langle x-x^{\prime}\right\rangle\right\} d V_{x^{\prime}},
\end{aligned}
$$

where $\beta$ is the reference configuration of the body, $\rho$ is the density in the reference configuration, $u$ is the displacement, and $b$ is the body force density. Equation (2) is a function of displacement that represents the internal force density (per unit volume) exerting on $x$ by other body points. Since force vector state $\underline{T}[x, t]$ depends only on the deformation of the family of $x$, and defining the vector $x^{\prime}-x=\xi$ to be a bond, we assume the following:

$$
|\xi|>\delta \Rightarrow T\langle\xi\rangle=0 .
$$

Under the assumption of a small deformation that does not need a small deformation gradient, the peridynamic equation (1) is accurately approximated by the following linear integrodifferential equation:

$$
\rho(x) \ddot{u}(x, t)=\int_{N_{x}} C(x, q)(u(q, t)-u(x, t)) d V_{q}+b(x, t),
$$

where $N_{x}$ is a neighborhood of $x$. As discussed in [20], the radius of $N_{x}$ is in general $2 \delta$, where $\delta$ is the horizon. $C(x, q)$ is a tensor-valued function which is named "the micromodulus function."

Similar to the linear classical theory, the linear peridynamic theory concerns small deformations. However, the damage and fracture that may be included in a linearized model, as noted above, does not require small displacement gradients [20]. Under this assumption of smallness, the peridynamic equation of equilibrium reduces to a linear integral equation. The linearized theory is more compatible with implicit solvers than the full theory. It is also much easier to obtain theoretical results about the properties of the linear equations such as well posedness than those of the nonlinear model. The properties of waves are also much easier to analyze within the linearized theory.

\section{Wave Dispersion in Linear Peridynamic Media}

The one-dimensional linear peridynamic wave model is popular because of its simplicity. Butt et al. [23] focused on the dispersion properties of a state-based linear peridynamic model. They derived the dispersion relation for one-, two-, and three-dimensional cases and specifically investigated the effect of the horizon, mesh size, and weighting function on the dispersion. Here, we focus on the dispersion characteristics of waves based on linear peridynamics. To study the peridynamic wave dispersion, we provide the general form of a plane wave:

$$
u(x, t)=A e^{i(k x-\omega t)} .
$$

Of these, $A$ is the vibration amplitude and $u$ is the displacement, with $k$ as the wavenumber and $w$ as the circular frequency. When the external force density in (4) is zero, we can obtain the following equation by substituting (5) into (4):

$$
-\omega^{2} \rho(x) u(x, t)=\int_{N_{x}} C(\xi)(u(q, t)-u(x, t)) d V_{q} .
$$

Here, $\xi=q-x$ is a bond between two material points. Changing the form of equation (6) leads to

$$
-\omega^{2} \rho(x) u(x, t)=\int_{N_{x}} C(\xi)\left(e^{i k \xi}-1\right) d V_{\xi} \cdot u(x, t) .
$$

The Euler transform in (7) is as follows:

$$
\begin{aligned}
\omega^{2} \rho(x) u(x, t)= & \int_{N_{x}} C(\xi)(1-\cos (k \xi)-i \sin (k \xi)) d V_{\xi} \\
& \cdot u(x, t) .
\end{aligned}
$$

The micromodulus function $C(\xi)$ is an even function, and $\sin (k \xi)$ is an odd function and integrated into the asymmetric domain $N_{x}$. Thus, we can get the following:

$$
\int_{N_{x}} C(\xi)(i \sin (k \xi)) d V_{\xi}=0
$$


Thus, we conclude from (8) and (9) that

$$
\omega^{2} \rho(x) u(x, t)=\int_{N_{x}} C(\xi)(1-\cos (k \xi)) d V_{\xi} \cdot u(x, t) .
$$

We conclude from equation (10) that the dispersion of the peridynamic wave is only related to the micromodulus function, density, and horizon. These parameters are inherent properties of the material.

The micromodulus function $C(\xi)=C(x, q)$ can be written as

$$
\begin{aligned}
C(x, q)= & \left(\frac{15 \mu}{m^{2}}-\frac{9 \kappa}{m^{2}}\right) \int_{H_{p}} \omega(|p-x|) \omega(|p-q|)(p-x) \\
& \otimes(p-q) d V_{p}+2 r(q-x), \\
& r(\xi)=\frac{15 \mu}{m} \omega(|\xi|)(M \otimes M) .
\end{aligned}
$$

Of these, $M=\xi /|\xi|, m=\int_{H} \omega(|\xi|)|\xi|^{2} d V_{\xi} \kappa$ is the bulk modulus, $\mu$ is the shear modulus, and $\omega$ is the weight function. The weight function and horizon selection are under intense investigation (equation (11)). If the selected horizon and weight function are consistent with the properties of the material, then the simulation results are close to the experimental results. Otherwise, a large error will occur. Equations (10) and (11) can lead to the following:

$$
\begin{aligned}
\omega^{2} \rho(x) u(x, t) & =M(k, \xi) u(x, t) \\
M(k, \xi) & =\int_{N_{x}} C(\xi)(1-\cos (k \xi)) d V_{\xi} .
\end{aligned}
$$

From (11) and (12), we found that $M(k, \xi)$ is a matrix (dispersion matrix). This matrix first appeared in [10].

This work assumes that a wave propagates along the wave vectors $n=[1,0,0]^{\mathrm{T}}$ in a Cartesian coordinate system. At this time, the stress wave propagates along $x_{1}$, which can be seen as a one-dimensional rod model. Thus, we can obtain $\xi=\xi_{1}$. The expansion of (13) is

$$
\omega^{2} \rho(x)\left(\begin{array}{l}
u_{1}(x, t) \\
u_{2}(x, t) \\
u_{3}(x, t)
\end{array}\right)=\left(\begin{array}{lll}
M_{11} & M_{12} & M_{13} \\
M_{21} & M_{22} & M_{23} \\
M_{31} & M_{32} & M_{33}
\end{array}\right)\left(\begin{array}{l}
u_{1}(x, t) \\
u_{2}(x, t) \\
u_{3}(x, t)
\end{array}\right) .
$$

When we study a longitudinal wave propagating along $x_{1}$, we can obtain only the displacement of $u_{1}(x, t) \neq 0$. Equation (15) leads to the following:

$$
\begin{aligned}
\omega_{11}^{2} \rho(x) u_{1}(x, t)=M_{11} u_{1}(x, t) \\
=\int_{N_{x}} C_{11}(\xi)\left(1-\cos \left(k \xi_{1}\right)\right) d V_{\xi} u_{1}(x, t) .
\end{aligned}
$$

Equation (16) shows that the longitudinal wave along the direction $x_{1}$ is related to the material parameter $C_{11}(\xi)$; thus, we can obtain the longitudinal wave dispersion equation by simplifying (16) to be

$$
\omega_{11}=\sqrt{\frac{1}{\rho(x)} \int_{N_{x}} C_{11}(\xi)\left(1-\cos \left(k \xi_{1}\right)\right) d V_{\xi}} .
$$

Similarly, the displacement of the horizontal shear wave propagating in the direction $x_{1}$ only has $u_{2}(x, t) \neq 0$. A combination of (14) and (15) shows that the dispersion is related to the material parameters $C_{22}(\xi)$. The dispersion equation is as follows:

$$
\omega_{12}=\sqrt{\frac{1}{\rho(x)} \int_{N_{x}} C_{22}(\xi)\left(1-\cos \left(k \xi_{1}\right)\right) d V_{\xi}}
$$

Similarly, as the displacement of the vertical shear wave propagates along $x_{1}$, only $u_{3}(x, t) \neq 0$. Combination of (14) and (15) shows that the dispersion is related to the material parameters $C_{33}(\xi)$. The dispersion equation is as follows:

$$
\omega_{13}=\sqrt{\frac{1}{\rho(x)} \int_{N_{x}} C_{33}(\xi)\left(1-\cos \left(k \xi_{1}\right)\right) d V_{\xi}}
$$

Equations (11) and (12), and the symmetry of the spatial integral indicate that the micromodulus function matrix in an infinite media is a symmetric matrix; the values on the diagonal are equal. Therefore, (18) and (19) indicate the same shear wave dispersion equation with only different vibration directions.

3.1. Longitudinal Wave Dispersion. This peridynamic method was used to simulate the wave dispersion in maraging steel. The following terms were used: material density $\rho(x)=8000 \mathrm{~kg} / \mathrm{m}^{3}$, bulk modulus $\kappa=1.59 \times 10^{11} \mathrm{pa}$, shear modulus $\mu=8.2 \times 10^{10}$ pa, mesh size $h=1 \mathrm{~mm}$, and horizon $\delta=3.015 h$. If there was a vibration at the center of the infinite media, then the longitudinal wave propagates along $x_{1}$ within the infinite media, and we can obtain the discrete equation from dispersion equation (17) as

$$
\omega_{11}=\sqrt{\frac{1}{\rho} \sum_{i=1}^{N_{x}} C_{11}\left(\xi_{i}\right)\left(1-\cos \left(k \xi_{i 1}\right)\right) \Delta V_{\xi_{i}}}
$$

where

$$
\begin{aligned}
C_{11}= & \left(\frac{15 \mu}{m^{2}}-\frac{9 \kappa}{m^{2}}\right) \sum_{j=1}^{p, j \neq i} \omega\left(\left|p_{j}-x\right|\right) \omega\left(\left|p_{j}-q_{i}\right|\right)\left(p_{j 1}-x_{1}\right) \\
& \cdot\left(p_{j 1}-q_{i 1}\right) \Delta V_{j}+\frac{30 \mu}{m} \omega(|\xi|)\left(\frac{q_{i 1}-x_{1}}{|\xi|}\right)^{2},
\end{aligned}
$$

$$
m=\sum_{k=1}^{H_{x}} \omega\left(\left|\xi_{k}\right|\right)\left|\xi_{k}\right|^{2} \Delta V_{\xi_{k}}
$$

The literature [5] shows that the relationship between the wavenumber, phase velocity, and circular frequency is as follows: 


$$
C_{\mathrm{p}}=\frac{\omega}{k}
$$

The group velocity can be found from the phase velocity via formula (24):

$$
C_{\mathrm{g}}=\frac{d \omega}{d k}
$$

Figures 1-3 plots the dispersion curve, phase velocity, and group velocity, respectively, from (20)-(24).

Figure 1 shows that the circular frequency of the longitudinal wave increases with the wavenumber, but the rate of circular frequency gradually decreases. Figure 2 shows that the phase velocity of the longitudinal wave reaches a maximum of $5539.8 \mathrm{~m} / \mathrm{s}$ when the wavenumber is near zero, which is consistent with the longitudinal wave velocity of $5500 \mathrm{~m} / \mathrm{s}$ in maraging steel. The phase velocity gradually decreases with increasing wavenumber. The group velocity is equal to the phase velocity when the wavenumber is near zero (Figure 3). At this point, there is no dispersion, but the longitudinal wave begins to disperse as the wavenumber increases, and the group velocity decays faster than the phase velocity.

The analysis above shows that the longitudinal wave dispersion is related to the material parameters. Equation (21) indicates that the material parameters are related to the weight function, horizon, and mesh size. Next, we separately study the effects on the dispersion curve. Given a material point $x$ at the origin, the radius of the horizon domain is $\delta=3.015 h$ and the mesh size is $h=1 \mathrm{~mm}$. The dispersion curves at different weight functions are shown in Figure 4.

In Figure 4, we see that the dispersion curve of the wave function is basically the same under the weight functions $\omega_{1}$ and $\omega_{2}$, but the rate of circular frequency of the dispersion curve is negative when the wavenumber is large. This indicates that the group velocity of the wave is negative, which does not match the actual situation. Therefore, the weight function $\omega_{3}$ is better for wave simulation when the wavenumber is large. The dispersion under the three weight functions is similar when the wavenumber is small, and the calculation is simple under $\omega_{2}$.

When the mesh size is $h=1 \mathrm{~mm}$, the weight function is $\exp \left(-\xi^{2} / \delta^{2}\right)$, and the radius of the horizon domain is $\delta=2 \mathrm{~mm}, 3 \mathrm{~mm}, 5 \mathrm{~mm}$, and $7 \mathrm{~mm}$. The dispersion image of the longitudinal wave is shown in Figure 5.

The horizon has less influence on dispersion when the wavenumber is small (Figure 5). When the wavenumber is large, the dispersion becomes more obvious as the radius of the horizon increases. When the radius of the horizon is $5 \mathrm{~mm}$ and $7 \mathrm{~mm}$, the group velocity is negative, which is inconsistent with the actual situation. The calculation is complicated in both cases. A horizon radius of $2 \mathrm{~mm}$ and $3 \mathrm{~mm}$ can better reflect the dispersion phenomenon of longitudinal waves.

When the horizon is $\delta=3 \mathrm{~mm}$, the weight function is $\exp \left(-\xi^{2} / \delta^{2}\right)$ and the mesh size is $h=1.5 \mathrm{~mm}, 1.0 \mathrm{~mm}$, $0.6 \mathrm{~mm}$, and $0.5 \mathrm{~mm}$. The dispersion curve of the longitudinal wave is shown in Figure 6.

Figure 6 shows that the mesh size has little effect on the dispersion when the wavenumber is small, but the mesh size

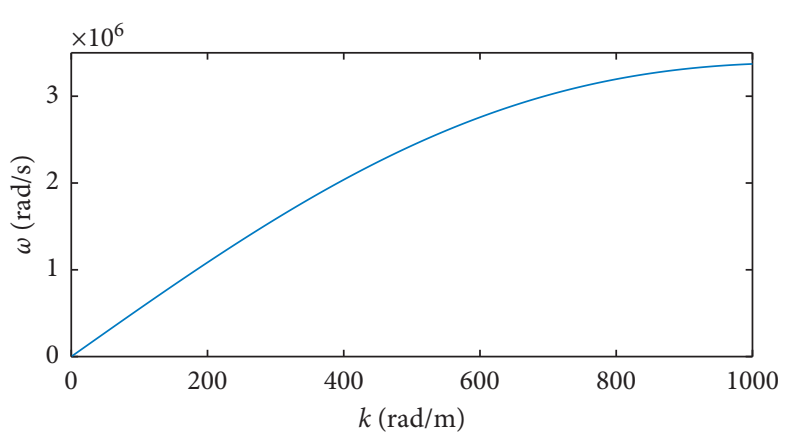

FIgURE 1: Longitudinal wave dispersion.

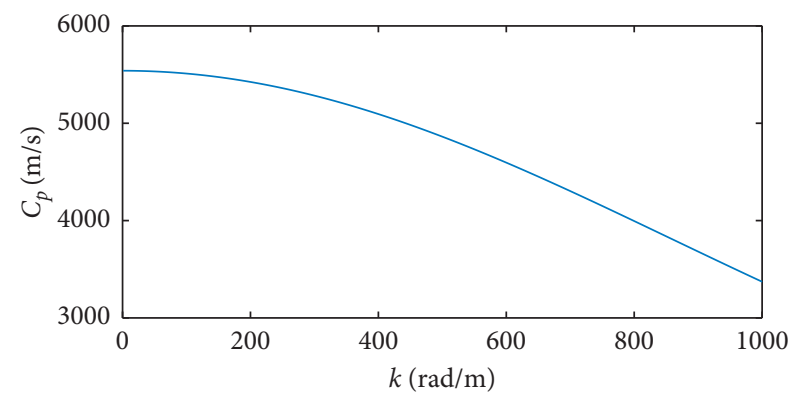

Figure 2: Phase velocity of longitudinal wave.

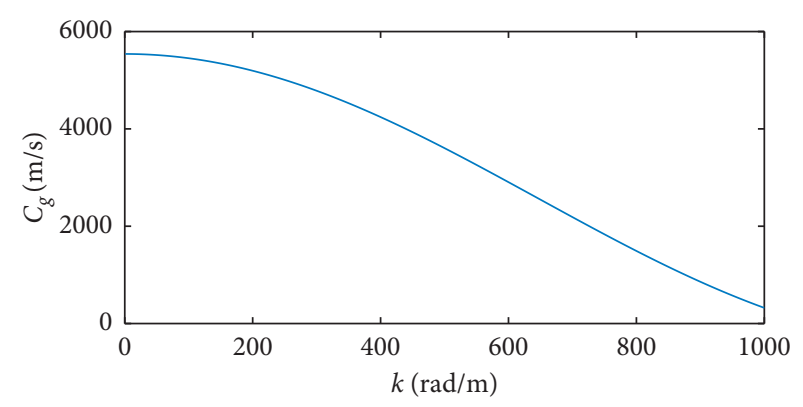

FIGURE 3: Group velocity of longitudinal wave.

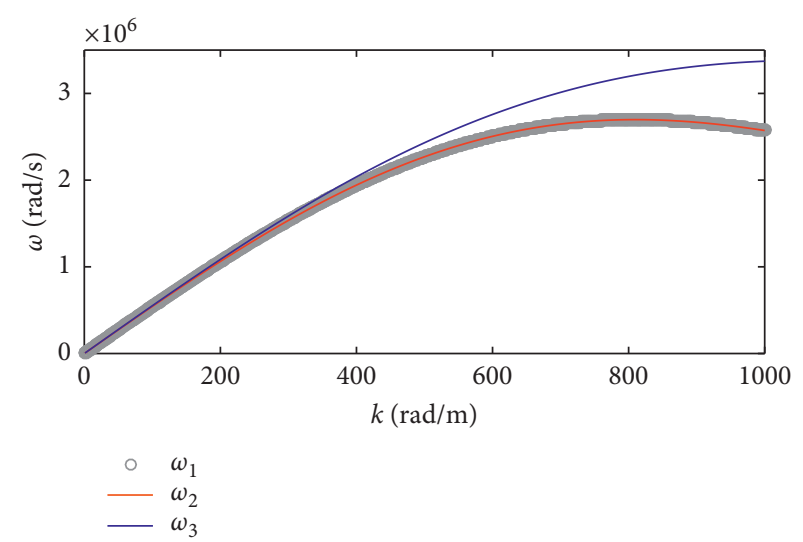

FIGURE 4: Longitudinal wave dispersion curve affected by a weight function.

is more pronounced when the wavenumber is large. When the mesh size is $h_{1}=1.5 \mathrm{~mm}$, the group velocity is negative, which is not consistent with the actual data. Although the 


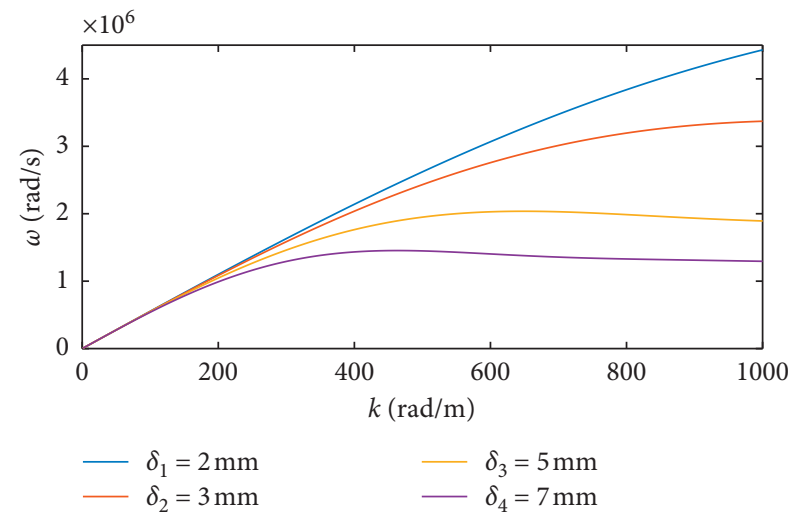

FIgURE 5: Longitudinal wave dispersion curve affected by the horizon.

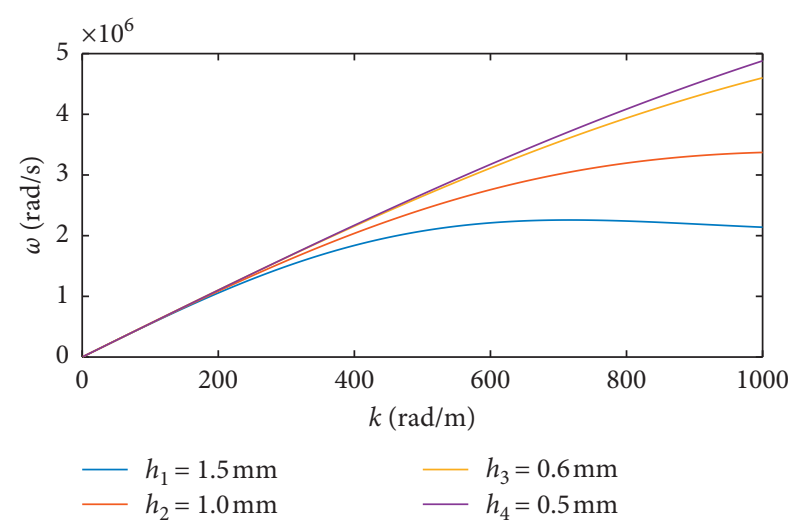

Figure 6: Longitudinal wave dispersion curve affected by mesh size.

dispersion is not obvious when $h_{3}=0.6 \mathrm{~mm}$ and $h=0.5 \mathrm{~mm}$, the computation is complex.

3.2. Shear Wave Dispersion. The material parameters are consistent with the longitudinal wave, and a shear wave propagates along the direction $x_{1}$ with a load at the center of the infinite media. The dispersion equation is indicated by (18) or (19). The two dispersion equations are consistent. By discretizing the dispersion equations, we can obtain

$$
\begin{aligned}
\omega_{12}= & \sqrt{\frac{1}{\rho} \sum_{i=1}^{N_{x}} C_{22}\left(\xi_{i}\right)\left(1-\cos \left(k \xi_{i 1}\right)\right) \Delta V_{\xi_{i}}} \\
C_{22}= & \left(\frac{15 \mu}{m^{2}}-\frac{9 \kappa}{m^{2}}\right) \sum_{j=1}^{p, j \neq i} \omega\left(\left|p_{j}-x\right|\right) \omega\left(\left|p_{j}-q_{i}\right|\right)\left(p_{j 2}-x_{2}\right) \\
& \cdot\left(p_{j 2}-q_{i 2}\right) \Delta V_{j}+\frac{30 \mu}{m} \omega(|\xi|)\left(\frac{q_{i 2}-x_{2}}{|\xi|}\right)^{2} .
\end{aligned}
$$

The term $m$ is given in (22).
From (22)-(24), the dispersion curve, phase velocity, and group velocity are given in Figures $7-9$, respectively.

Figures 7-9 indicate that the properties of the shear wave such as dispersion and wave velocity are similar to those of the longitudinal wave. The circular frequency increases with the wavenumber, but the rate of circular frequency gradually decreases. The phase velocity and group velocity of the shear wave reach $3216.4 \mathrm{~m} / \mathrm{s}$ when the wavenumber nears zero. This agrees with the shear wave velocity of $3200 \mathrm{~m} / \mathrm{s}$ in maraging steel. As the wavenumber increases, the phase velocity gradually slows down and starts to disperse and the group velocity decays faster than the phase velocity.

Through the above analysis, it can be observed that the dispersion of the shear wave and the longitudinal wave is affected by similar material parameters, such as the weight function, horizon, and mesh size. Given a material point $x$ at the center of the origin, the radius of the horizon domain is $\delta=3.015 h$ and the mesh size is $h=1 \mathrm{~mm}$, the dispersion curves at different weight functions are shown in Figure 10.

When the mesh size is $h=1 \mathrm{~mm}$, the weight function is $\exp \left(-\xi^{2} / \delta^{2}\right)$ and the radius of the horizon domain is $\delta=2 \mathrm{~mm}, 3 \mathrm{~mm}, 5 \mathrm{~mm}$, and $7 \mathrm{~mm}$; the dispersion of the shear wave is shown in Figure 11.

When the horizon is $\delta=3 \mathrm{~mm}$, the weight function is $\exp \left(-\xi^{2} / \delta^{2}\right)$ and the mesh sizes are $h=1.5 \mathrm{~mm}, 1.0 \mathrm{~mm}$, $0.6 \mathrm{~mm}$, and $0.5 \mathrm{~mm}$; the dispersion curve of the shear wave is shown in Figure 12.

Figures 10-12 indicate that the dispersion of the shear wave is influenced by the weight function, horizon, and mesh size. It is the same as the longitudinal wave. The dispersion curve is roughly the same under the three weight functions, except that the dispersion of the wave function is more pronounced under the weight functions $\omega_{1}$ and $\omega_{2}$. When the wavenumber is low, the horizon has little effect on the dispersion. When the wavenumber is large, the dispersion becomes more obvious with horizon increases. A small mesh size leads to a more obvious dispersion. However, the mesh size is relatively small when the horizon is large, and there are more material points involved in the horizon-this increases the complexity of the calculation.

Figures 4 and 10 show that the dispersion with different weight functions has little difference when the wavenumber is small. However, when the wavenumber is around $1000 \mathrm{rad} / \mathrm{m}$, the group velocity becomes negative with weight functions $\omega_{1}$ and $\omega_{2}$. This indicates that the wave propagates in the inverse direction; this phenomenon does not occur in the real world. Thus, the weight function $\omega_{3}$ is better for simulation. Figures 5 and 11 show that the dispersion is noticeable as the horizon domain increases. The wave group velocity becomes negative when the horizon is greater than three times the mesh sizes-especially in the longitudinal wave. The peridynamic wave equation will converge to the classical Navier equation when the horizon is small. The horizon should be selected between two and three times the mesh sizes. In Figures 6 and 12, we see that the dispersion is weakened with decreasing mesh 


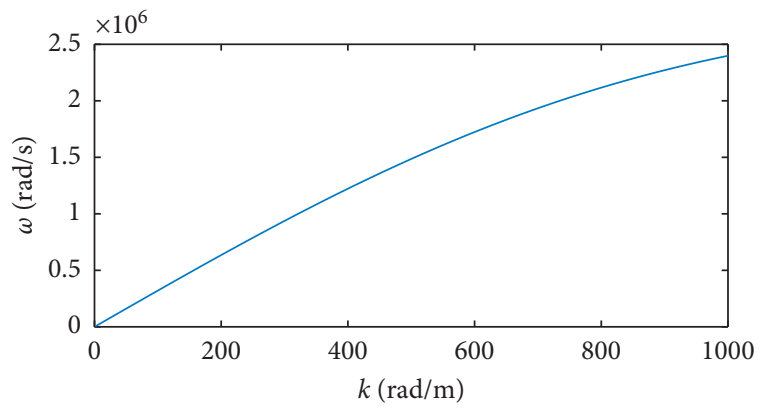

Figure 7: Shear wave dispersion.

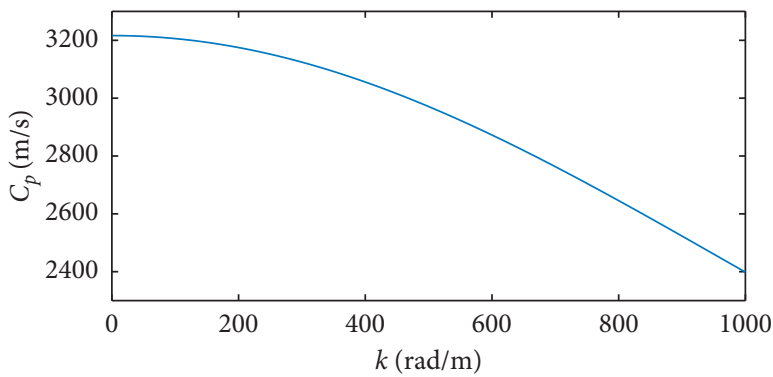

FIgUre 8: Phase velocity of the shear wave.

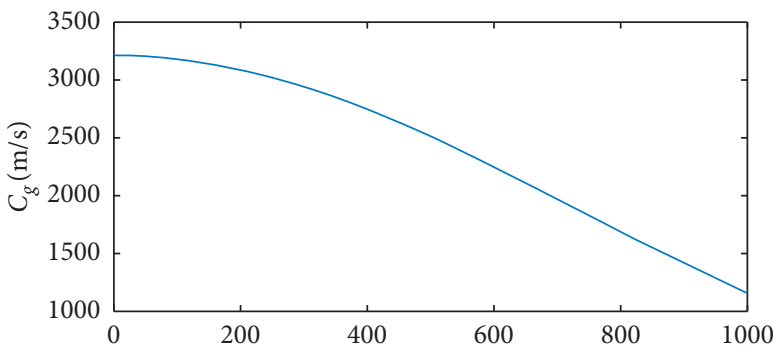

Figure 9: Group velocity of the shear wave.

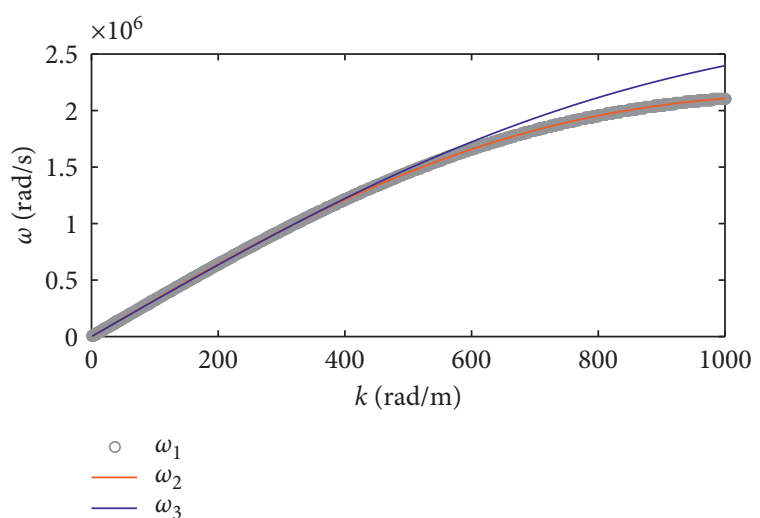

FIGURE 10: Shear wave dispersion curve affected by the weight function.

size. When the mesh size is small, there will be more material points on the horizon to complicate the calculations. The analysis above shows that the horizon should

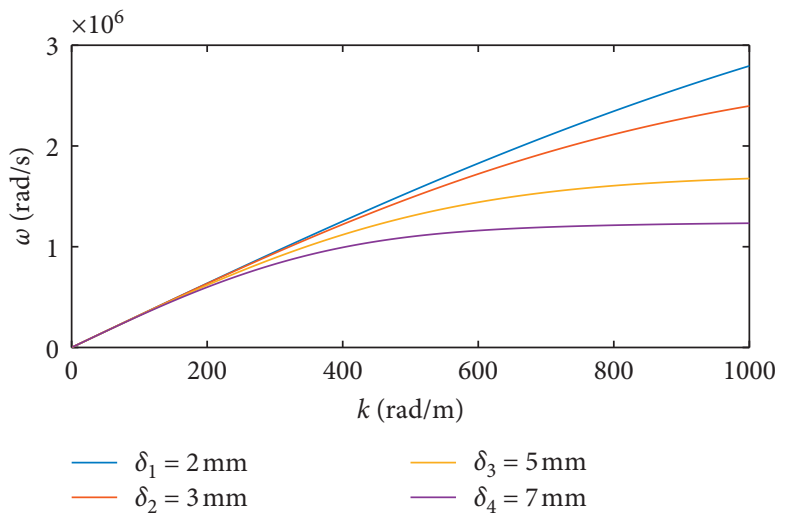

Figure 11: Shear wave dispersion curve affected by the horizon.

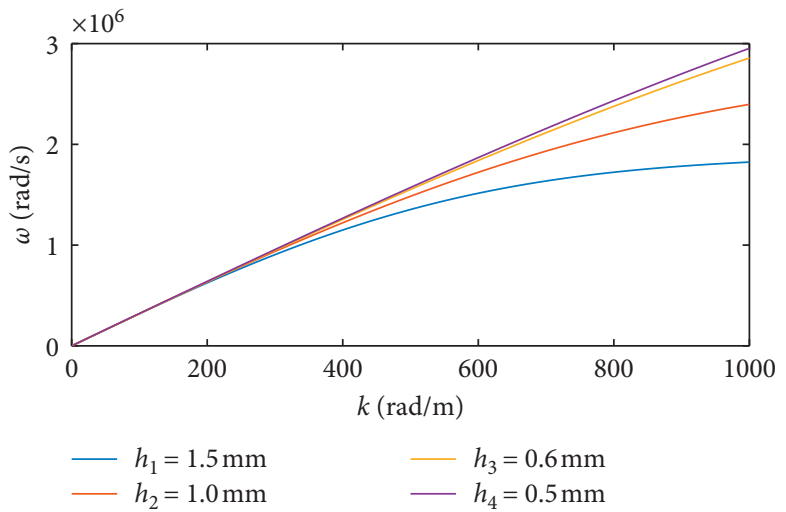

FIGURE 12: Shear wave dispersion curve affected by the mesh size.

be three times the mesh sizes [24]. Therefore, in this paper, the weight function should be $\exp \left(-\xi^{2} / \delta^{2}\right)$, the horizon should be $\delta=3 \mathrm{~mm}$, and the mesh size should be $h=1 \mathrm{~mm}$. This makes the wave simulation agree with natural reality.

\section{Numerical Simulation of Peridynamic Wave}

In order to study the ultrasonic wave propagation in an infinite media, we studied a cubic piece of maraging steel with the following properties: $0.1 \mathrm{~m} \times 0.1 \mathrm{~m} \times 0.1 \mathrm{~m}$, material density $\rho(x)=8000 \mathrm{~kg} / \mathrm{m}^{3}$, bulk modulus $\kappa=1.59 \times 10^{11} \mathrm{pa}$, shear modulus $\mu=8.2 \times 10^{10} \mathrm{pa}$, mesh size $h=1 \mathrm{~mm}$, horizon radius $\delta=3.015 h$, and time step $\Delta t=10^{-8} \mathrm{~s}$. A displacement of $u_{y}=10^{-10} \cos (-760000 \pi t)$ was placed along $y$ at the center of the cube. Based on the peridynamic simulation method [25], the displacement in direction $y$ on the $x o y$ plain can be obtained in Figure 13.

When the displacement load is applied in the direction $y$ at the center of the cube maraging steel material on the surface $x o y$, the load generated a longitudinal wave that propagates in the direction $y$. The shear wave propagates in the direction $x$. The other directions are superpositions of the two waveforms. By analyzing the waveform at $t=5 \mu \mathrm{s}$ and $t=10 \mu \mathrm{s}$ in Figure 13, we can obtain the wave 


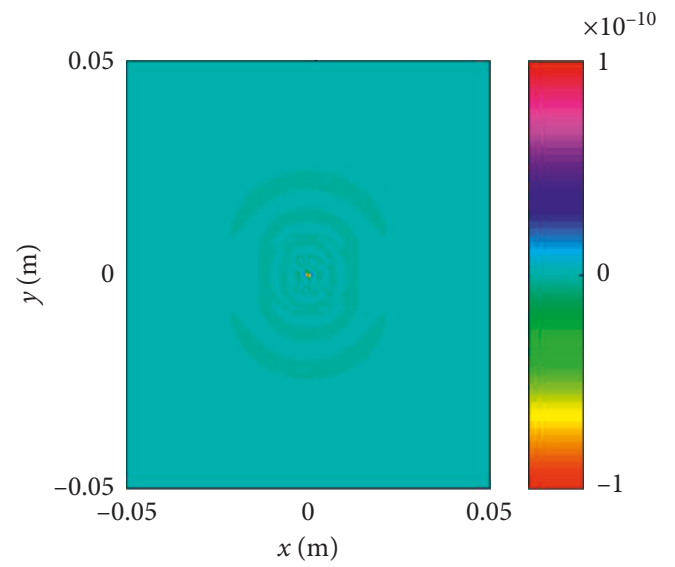

(a)

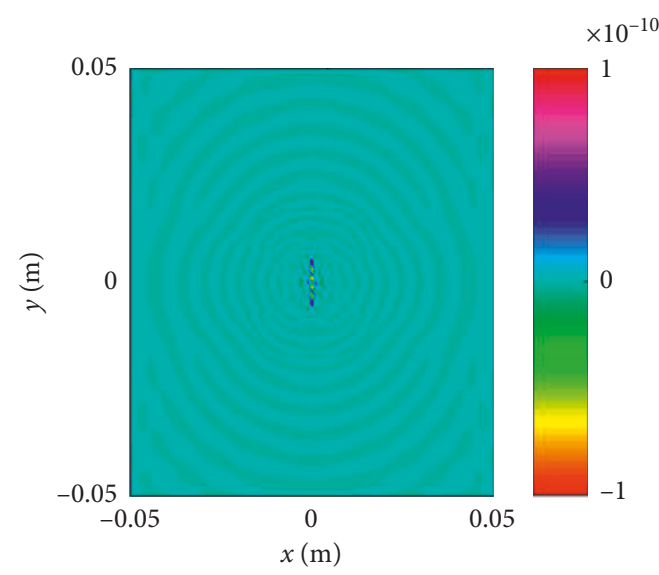

(c)

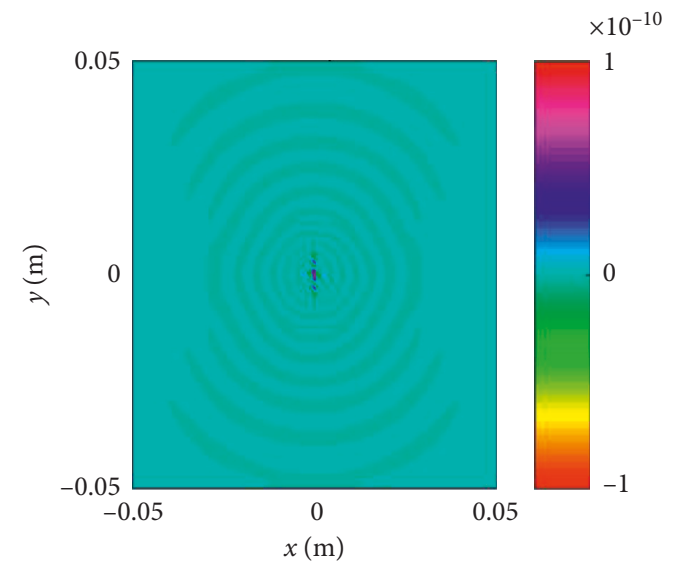

(b)

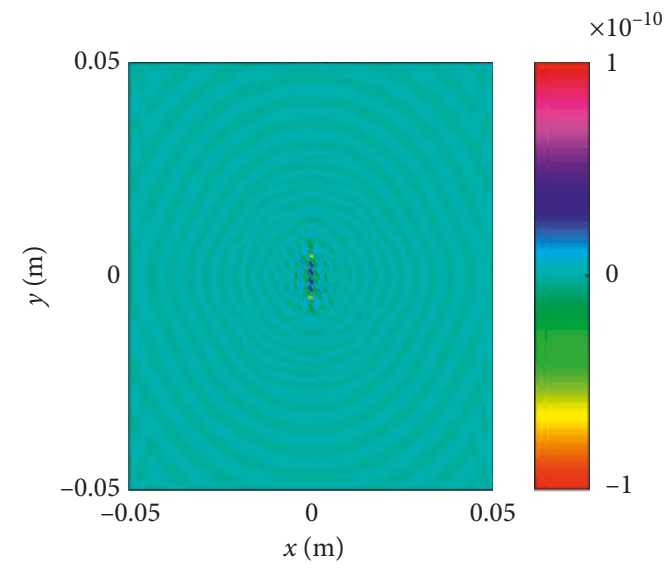

(d)

FIGURE 13: Displacement of a material point in direction $y$ on the surface $x o y$. (a) $t=5 \mu \mathrm{s}$. (b) $t=10 \mu \mathrm{s}$. (c) $t=15 \mu \mathrm{s}$. (d) $t=20 \mu \mathrm{s}$.

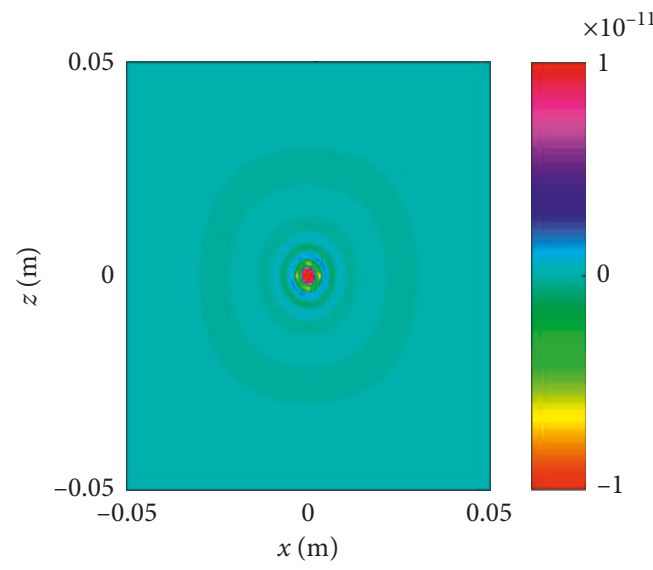

(a)

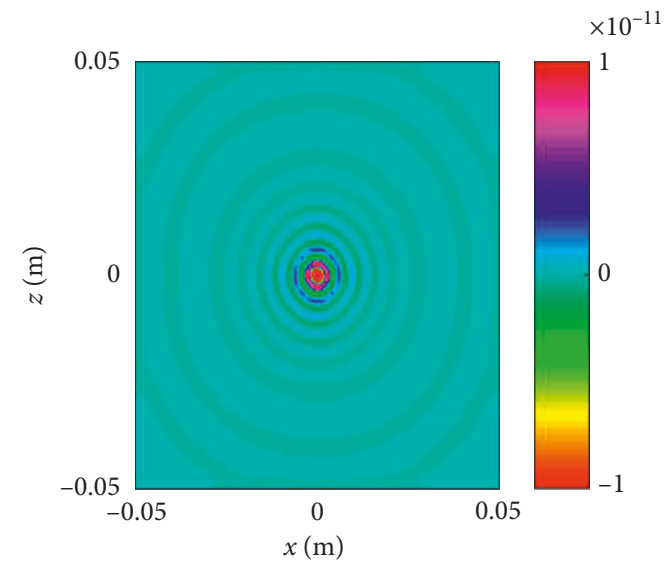

(b)

Figure 14: Vibration on the $x o z$ plane at different times. (a) $t=5 \mu \mathrm{s}$. (b) $t=10 \mu \mathrm{s}$.

propagation in the direction $y$. It is faster than that in the direction $x$, which is consistent with the observation that the velocity of the longitudinal wave is greater than that of the shear wave. Thus, the wavefront on the xoy surface is an elliptical surface. When $t=15 \mu \mathrm{s}$ in Figure 13, the waveform is reflected with the free boundary leading to a waveform transform; the incident wave and the reflected wave are superimposed resulting in the wavelength shortening and an increase in the wavenumber. This phenomenon is even more pronounced when $t=20 \mu \mathrm{s}$ in Figure13. 
Ultrasonic waves propagate in all directions in the cube. The two different shear waves can be obtained at the same time on the surface $x o z$, and they are both displaced in the direction $y$. These two shear wave vibrations are shown in Figure 14.

The ultrasonic waves propagate in the directions $x$ and $z$ with the same wave velocity when given a load in the direction $y$. Figure 14 shows that the vibrations in each direction on $x o z$ are basically the same; the wavefront is a circular surface. The wave propagates in an infinite media, and the longitudinal wave propagates along the $y$-axis. Here, the horizontal shear wave propagates along the $x$-axis, and the vertical shear wave propagates along the $z$-axis. The wavefront in the infinite media is an ellipsoid because the longitudinal wave and shear wave have different velocities.

\section{Conclusions}

In this paper, the integral equations for the wave are given by the peridynamic theory. By studying the linearization of the wave peridynamic equation, the dispersions of the longitudinal and shear waves are given in the infinite media. The phase velocity and the group velocity of the wave are obtained, which are consistent with the wave velocity in classical mechanics. The dispersion curve is influenced by the weight function, horizon, and mesh size. When the weight function is $\exp \left(-\xi^{2} / \delta^{2}\right)$, the horizon is three times the mesh size and the dispersion curve can respond to the wave dispersion in the maraging steel. It is better than the dispersion curve with other parameters. The peridynamic theory evaluated the numerical simulation of the wave. Under initial loads at direction $y$, we found that the longitudinal wave was excited in the direction of $y$, which on the other main axis was a shear wave. The longitudinal and shear wave have different wave velocities, and thus the wavefront in the cube is an ellipsoid.

\section{Data Availability}

The data used to support the findings of this study are available from the corresponding author upon request.

\section{Conflicts of Interest}

The authors declare that they have no conflicts of interest.

\section{Acknowledgments}

The work was supported by the National Natural Science Foundation of China (No. 51679112) and Postgraduate Research \& Practice Innovation Program of Jiangsu Province KYCX17_1764. The authors thank LetPub (http://www. letpub.com) for its linguistic assistance during the preparation of this manuscript.

\section{References}

[1] D. V. Achillopoulou and A. Pau, "Characterization of defects in plates using shear and Lamb waves," Procedia Engineering, vol. 199, pp. 2001-2007, 2017.
[2] Y. Cui, Suudy on Lamb Waves Propagation Characteristics in Plates with Butt Welds and Inspection of Defects, Beijing University of Technology, Beijing, China, 2014.

[3] Z. Fan and M. J. S. Lowe, "Interaction of weld-guided waves with defects," NDT \& E International, vol. 47, pp. 124-133, 2012.

[4] Z. Fan and M. J. Lowe, "Elastic waves guided by a welded joint in a plate," Proceedings of the Royal Society of London A: Mathematical, Physical and Engineering Sciences, vol. 465, no. 2107, pp. 2053-2068, 2009.

[5] J. L. Rose, Ultrasonic Guided Waves in Solid Media, Cambridge University Press, Cambridge, UK, 2014.

[6] A. Fabro, N. Ferguson, and B. Mace, "Wave propagation in slowly varying waveguides using a finite element approach," Journal of Sound and Vibration, vol. 442, pp. 308-329, 2019.

[7] J.-M. Mencik, "A wave finite element approach for the analysis of periodic structures with cyclic symmetry in dynamic substructuring," Journal of Sound and Vibration, vol. 431, pp. 441-457, 2018.

[8] M. Cong, X. Wu, and R. Liu, "Dispersion analysis of guided waves in the finned tube using the semi-analytical finite element method," Journal of Sound and Vibration, vol. 401, pp. 114-126, 2017.

[9] K. Hellan, Introduction to Fracture Mechanics, McGraw-Hill, New York, NY, USA, 1984.

[10] S. A. Silling, "Reformulation of elasticity theory for discontinuities and long-range forces," Journal of the Mechanics and Physics of Solids, vol. 48, no. 1, pp. 175-209, 2000.

[11] E. Madenci and E. Oterkus, Peridynamic Theory and its Applications, Springer, Berlin, Germany, 2014.

[12] M. Zingales, "Wave propagation in 1D elastic solids in presence of long-range central interactions," Journal of Sound and Vibration, vol. 330, no. 16, pp. 3973-3989, 2011.

[13] K. Kiani, "Small-scale effect on the vibration of thin nanoplates subjected to a moving nanoparticle via nonlocal continuum theory," Journal of Sound and Vibration, vol. 330, no. 20, pp. 4896-4914, 2011.

[14] A. C. Eringen and D. G. B. Edelen, "On nonlocal elasticity," International Journal of Engineering Science, vol. 10, no. 3, pp. 233-248, 1972.

[15] A. C. Eringen, C. G. Speziale, and B. S. Kim, "Crack-tip problem in non-local elasticity," Journal of the Mechanics and Physics of Solids, vol. 25, no. 5, pp. 339-355, 1977.

[16] S. A. Silling and F. Bobaru, "Peridynamic modeling of membranes and fibers," International Journal of Non-Linear Mechanics, vol. 40, no. 2-3, pp. 395-409, 2005.

[17] S. A. Silling, M. Zimmermann, and R. Abeyaratne, "Deformation of a peridynamic bar," Journal of Elasticity, vol. 73, no. 1-3, pp. 173-190, 2003.

[18] M. Zimmermann, A continuum theory with long-range forces for solids, Ph.D thesis, Massachusetts Institute of Technology, Cambridge, MA, USA, 2005.

[19] S. A. Silling, M. Epton, O. Weckner, J. Xu, and E. Askari, "Peridynamic states and constitutive modeling," Journal of Elasticity, vol. 88, no. 2, pp. 151-184, 2007.

[20] S. A. Silling, "Linearized theory of peridynamic states," Journal of Elasticity, vol. 99, no. 1, pp. 85-111, 2010.

[21] Z. P. Bažant, W. Luo, V. T. Chau, and M. A. Bessa, "Wave dispersion and basic concepts of peridynamics compared to classical nonlocal damage models," Journal of Applied Mechanics, vol. 83, no. 11, article 111004, 2016.

[22] S. A. Silling, "Solitary waves in a peridynamic elastic solid," Journal of the Mechanics and Physics of Solids, vol. 96, pp. 121-132, 2016. 
[23] S. N. Butt, J. J. Timothy, and G. Meschke, "Wave dispersion and propagation in state-based peridynamics," Computational Mechanics, vol. 60, no. 5, pp. 725-738, 2017.

[24] F. Bobaru, J. T. Foster, P. H. Geubelle, and S. A. Silling, Handbook of Peridynamic Modeling, CRC Press, Boca Raton, FL, USA, 2016.

[25] S. A. Silling, "Dynamic fracture modeling with a meshfree peridynamic code," in Proceedings of the Computational Fluid \& Solid Mechanics, pp. 641-644, Cambridge, MA, USA, June 2003. 


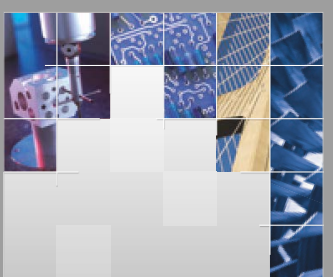

\section{Enfincering}
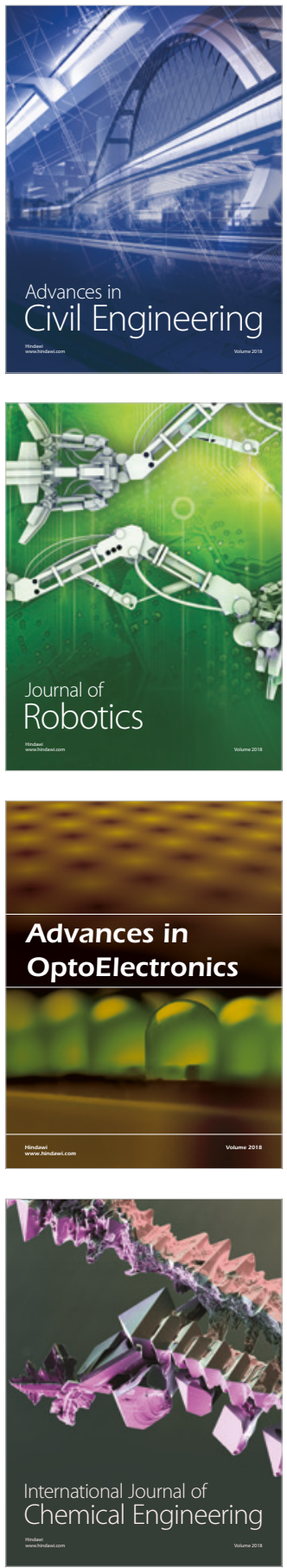

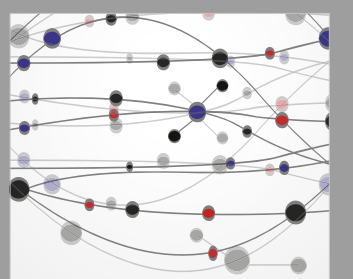

\section{Rotating \\ Machinery}

The Scientific World Journal

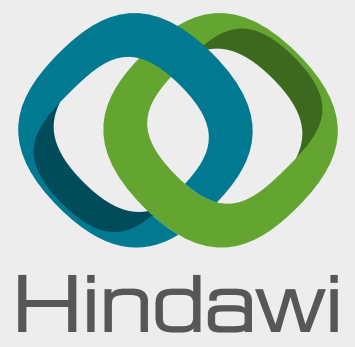

Submit your manuscripts at

www.hindawi.com
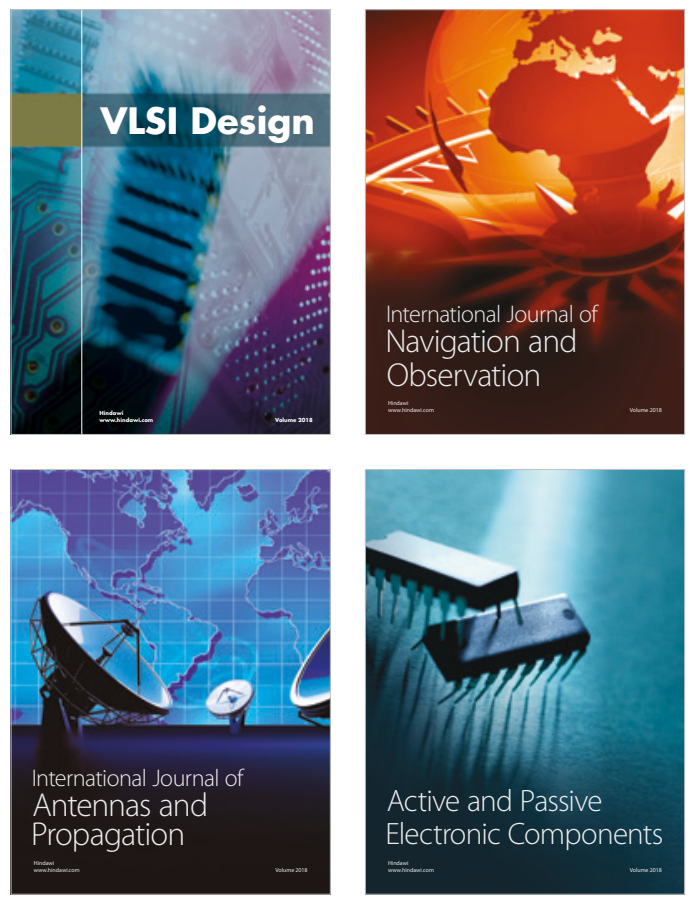
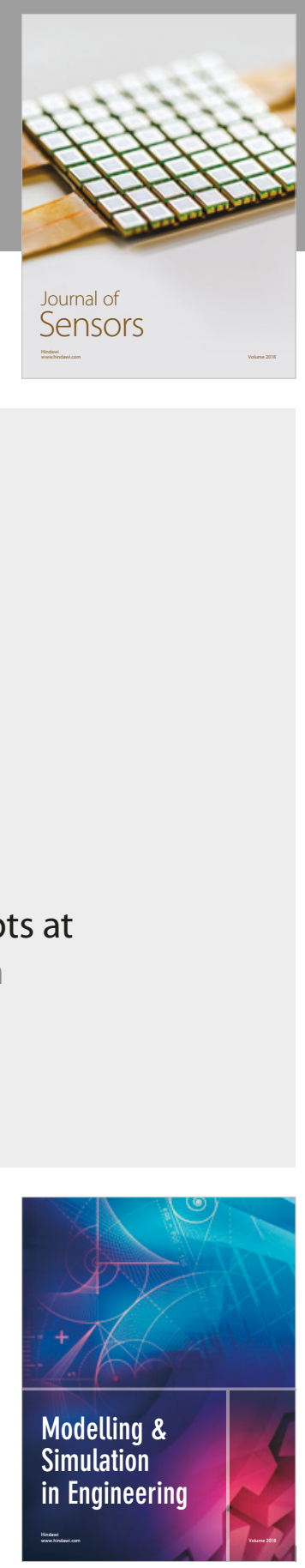

\section{Advances \\ Multimedia}
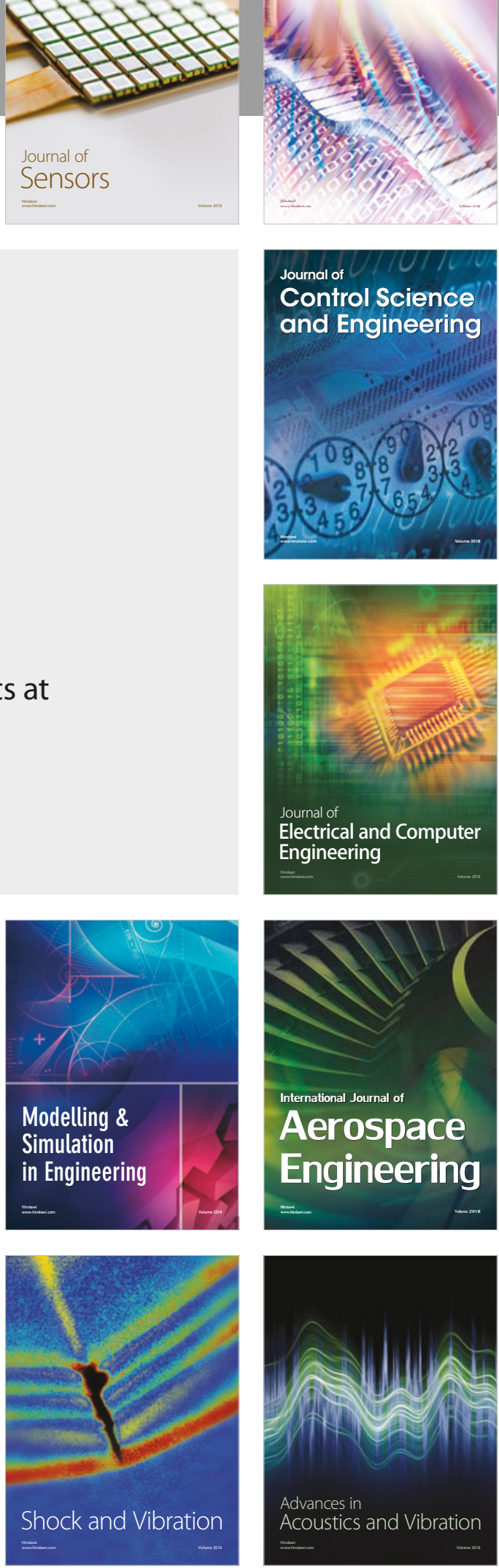